\title{
Nanopartikel am Arbeitsplatz
}

\author{
K. Schmid, B. Danuser und M. Riediker
}

Institut für Arbeit und Gesundheit, Lausanne

\begin{abstract}
Schlüsselwörter
Nanopartikel - Messge-

räte - Industrie - Ar-

beitsplatzanalyse
\end{abstract}

\section{Key words}

nanoparticles - measurement devices - industry - workplace assessment

\section{Nanopartikel am Arbeitsplatz}

Nanopartikel sind sehr kleine Partikel, die gezielt so hergestellt sind, dass ihr Durchmesser kleiner als etwa $100 \mathrm{~nm}$ ist [1]. Sie werden in der Industrie eingesetzt, weil Materialien mit solch kleinen Dimensionen oft neue Eigenschaften aufweisen, die sie vom Ursprungsmaterial unterscheidet. Das Potenzial für mögliche Gesundheits- und Umwelteffekte von Nanomaterialien wird zurzeit intensiv diskutiert, denn die möglichen Effekte der neuen Eigenschaften auf Umwelt und Gesundheit sind erst unvollständig geklärt. Für die Abklärung der Risiken ist es wichtig, Informationen über die möglichen Expositionen und mögliche Freisetzungen in die Umwelt zu haben. Bisher wurden aber Daten über eingesetzte Stoffmengen und Materialarten selten systematisch erhoben. Wir haben in der Schweiz eine repräsentative Studie durchgeführt, um den Einsatz von Nanopartikeln im gesamten Industriesektor abschätzen zu können. Diese Studie ist unseres Wissens weltweit die erste solche Studie. Sie verwendete die Definition von Nanopartikeln, welche Nanofasern und Agglomerate von Nanopartikeln mit einschließt. Geschätzte 1.300 Arbeiter in 600 Firmen sind direkt an einer Nanopartikelanwendung beteiligt und könnten somit exponiert werden. Dies sind etwa $0,6 \%$ der Firmen und etwa $0,08 \%$ der Arbeiter des Schweizer Produktionssektors. Um nun zu bestimmen, ob solche Arbeiter mit Nanopartikel in Kontakt kommen oder nicht, stehen verschiedene Messmethoden zur Verfügung. Die aktuelle Technik erlaubt eine quantitative Messung der Anzahl der Partikel in der Luft, deren Masse oder auch Oberfläche. Diese Messgrößen allein geben zwar Hinweise auf die Präsenz von Nanopartikeln, die möglichen Gesundheitseffekte einer Exposition sind aber erst unvollständig abgeklärt und erlauben keine abschließende Risikoanalyse für den Arbeitsplatz. Mehrere Aktionspläne für die Entwicklung eines sicheren und nachhaltigen Umgangs mit Nanomaterialien wurden in den letzten Jahren gestartet (EU, Schweiz). Internationale und nationale Organisationen entwickelten Guidelines und Empfehlungen für industrielle Anwendungen (Internationale Organisation für Nor- mung - ISO, Schweizerische Unfallversicherungsanstalt - SUVA, Bundesanstalt für Arbeitsschutz und Arbeitsmedizin - BAuA, zusammen mit dem Verband der Chemischen Industrie - VCI). Diese generellen Informationen müssen nun in die Industrie transferiert und an die spezifischen Bedürfnisse der betroffenen Unternehmen angepasst werden. Die aufgezeigte, relativ geringe Verbreitung von Nanopartikelanwendungen in der Industrie weist darauf hin, dass heute Schutzmaßnahmen noch proaktiv und kostengünstig entwickelt und eingeführt werden können. Aber sollte die vorhergesagte "Nano-Revolution" wirklich eintreten, ist die Zeit gekommen, jetzt aktiv zu werden.

\section{Nanoparticles at the workplace}

Nanoparticles have a diameter smaller than 100 nanometres in all three dimensions [1]. They are used in industry due to size specific properties, but the relevance of their potentially negative health effects is still insufficiently known. Addressing the risks of nanoparticles requires knowledge about occupational exposure and liberation into the environment. However, currently such information is not sufficiently available, as it is not systematically collected. The risk assessment lacks quantitative data in following crucial domains: the unknown number of exposed people and the level of their exposure or liberation of nanomaterials into the environment. We estimated the current usage of nanoparticles in the Swiss industry with a representative survey. To our knowledge this is the first such representative assessment for a country ever done. The definition of nanoparticles used for the survey includes fibres and agglomerates. Estimated 1,300 workers are potentially exposed to nanoparticles in 600 companies corresponding to $0.6 \%$ of companies in the producing sector. For the assessment of a workplace exposure to airborne nanoparticles, different techniques are available. The currently used technique allows quantitative measurements: the number, the mass or the surface of nanoparticles in the air. However, as the effects of nanoparticles on 
health and environment are not yet fully understood, the information gained by measurement allows not yet a comprehensive risk assessment for the occupational setting. Several action plans for the development of a safe usage of nanomaterials were set up in the last years to encounter these problems (EU, Switzerland). International and national organizations developed recommendations and guidelines for the industry (International Organization for Standardization - ISO, Swiss National Accident Insurance Fund - SUVA, German Federal Institute for Occupational Safety and Health - BAuA together with the German Chemical Industry Association VCI). The information in these guidelines needs to be transferred into the reality and adapted to the specific needs of each company and each work place. Noting the low nanoparticle usage, there still seems to be time to proactively introduce protection methods. But if the predicted "nano-revolution" becomes true, now is the time to take action.

\section{Einleitung}

Die Nanotechnologie wird oft als diejenige Entwicklung gepriesen, welche das 21. Jahrhundert prägen wird. Weltweit sind bereits mehr als 800 Nanoprodukte in der Konsumgüterdatenbank des Woodrow Wilson International Center for Scholars aufgelistet [2]. Die Liste wächst stetig an und verdreifachte sich in den letzten 2 Jahren. Bei all der Preisung der Vorzüge dieser Technologie wird aber erst seit kürzerer Zeit das Potenzial für mögliche Gesundheits- und Umwelteffekte diskutiert. Der Begriff Nanotechnologie ist sehr breit gefasst. Für die Gesundheitsdiskussion relevant sind längst nicht alle Bereiche der Nanotechnologie. Es ist vor allem die Herstellung oder Nutzung von Nanopartikeln (3 Dimensionen kleiner $100 \mathrm{~nm}$ ) und -fasern (2 Dimensionen kleiner $100 \mathrm{~nm}$ ) sowie deren Agglomerate, welche mit potenziellen Risiken in Verbindung gebracht werden. Solche Nanoobjekte werden verwendet, weil sie chemische, biologische oder physikalische Eigenschaften aufweisen, die anders sind als die Eigenschaften größerer Objekte der gleichen Substanz. Diese neuen Eigenschaften bieten zwar Chancen, aber auch Risiken.

Nanopartikel werden nicht nur für die Herstellung von Nanoprodukten verwendet, die klar deklariert sind, sondern auch in Pro- dukten, in welchen dies nicht offensichtlich ist. Vor der Einführung nanotechnologischer Alltagsprodukte ist aber eine gründliche Abklärung möglicher Risiken sehr wichtig. Die Risikodiskussion um synthetische Nanomaterialien dreht sich insbesondere um freie $\mathrm{Na}$ nopartikel und um Materialien, aus welchen solche freigesetzt werden können. Es ist wichtig zu unterscheiden, ob es sich tatsächlich um freie oder aber um gebundene Nanopartikel handelt (z.B. fest in einer Oberfläche eingebundene Partikel). Bei der gebundenen Form ist die Möglichkeit zum Kontakt oder zur Aufnahme in den Menschen stark reduziert. Bei freien Nanopartikeln oder deren Agglomeraten wiederum kommt es darauf an, ob sie in Flüssigkeit dispergiert oder lose als Pulver vorliegen. Weitere wichtige Faktoren sind die Materialzusammensetzung, die gesamte Oberfläche und die Reaktivität. In gewissen Fällen kann auch die Form der Partikel wichtig sein, wie zum Beispiel bei Kohlenstoffröhrchen (Carbon Nanotubes), die im Verdacht stehen, asbestähnliche Gesundheitseffekte auslösen zu können, wenn sie steif und ähnlich lang sind wie Asbestfasern.

Der Arbeitsplatz ist jener Bereich, in welchem Nanopartikel bereits heute von Bedeutung für die Gesundheit von Menschen sein können, denn in der Industrie werden vielfältige Einsatzmöglichkeiten von Nanopartikeln getestet und neue Produktionstechnologien entwickelt. Es ist zu erwarten, dass am Arbeitsplatz die ersten und deutlichsten Expositionen von Personen gegenüber Nanopartikel vorkommen. Hier werden zurzeit auch die höchsten Mengen erwartet, welche an die Umwelt abgegeben werden könnten.

Dieser Artikel gibt einen Überblick über die im Bereich Nanopartikel und Gesundheit aktiven nationalen (Schweiz/Deutschland) und internationalen (EU) Organe. Er fasst zusammen, mit welchen Messtechniken und Limits heute die Belastung von Arbeitsplätzen mit Nanopartikeln erfasst werden kann.

\section{Nanoinventar Schweiz}

Bis heute wurde noch in keinem Land systematisch untersucht, wie viele Unternehmen der gesamten (auch nanotechnologiefernen) Wirtschaft in ihrer Produktion Nanopartikel einsetzen, um welche Arten von Nanoparti- 


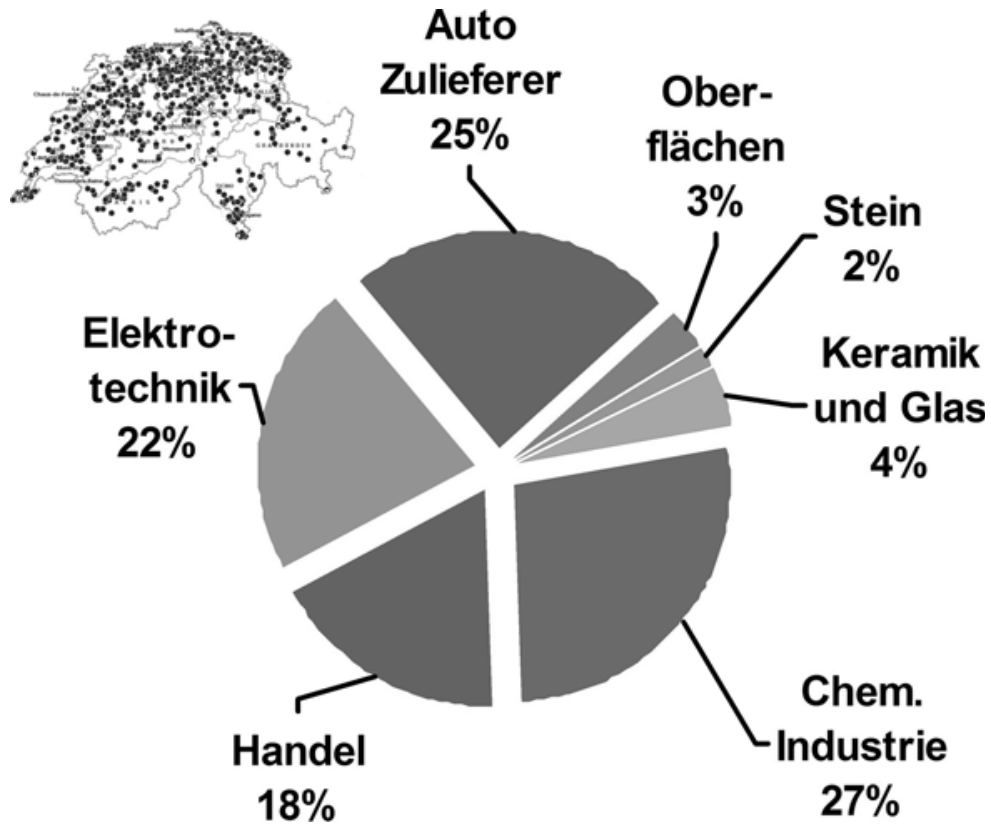

Abb. 1. Weniger als $1 \%$ der Schweizer Firmen des produzierenden Sektors wenden Nanopartikel an. Der Graph zeigt die Verteilung von Anwendungen innerhalb dieser Unternehmen mit Nanopartikeln.

keln es sich dabei handelt und in welchen Mengen diese eingesetzt und gelagert werden. Studien über industrielle Expositionen von Arbeitnehmern sind immer noch rar und die wenigen bereits getätigten Untersuchungen genügen nicht für eine systematische Risikoabschätzung der potenziellen Gefahren, welche für die Arbeitnehmer vom Kontakt mit Nanopartikeln während Herstellungsoder Verarbeitungsprozessen in der Industrie ausgehen können. Angaben zu umgesetzten und gelagerten Mengen sind kaum vorhanden, diese wären aber wichtig für eine $\mathrm{Ab}$ schätzung der möglichen Umweltrelevanz einzelner Nanomaterialarten.

Vor diesem Hintergrund wurde die Schweizer Studie "Nanoinventar" durchgeführt [3]. Sie sollte untersuchen, in welchen Mengen Nanopartikel bereits in der Schweizer Industrie eingesetzt und gelagert werden. Es galt abzuschätzen, wie viele Arbeitnehmer damit potenziell in Kontakt kommen können und welche Schutzstrategien für die Arbeitnehmer bzw. für die Umwelt an diesen Arbeitsplätzen angewendet werden. Der in dieser Studie verwendete Begriff "Nanopartikel" umfasst alle Partikel mit einem Durchmesser von 1-1.000 nm, also auch Agglomerate von Nanopartikeln und Nanofasern.
Die repräsentative Umfrage wurde hochgerechnet auf den Schweizer Industriesektor und zeigte, dass ca. 600 Unternehmen eine Nanopartikelanwendung aufweisen. In diesen Firmen arbeiten ca. 1.300 Personen direkt mit einer Nanopartikelanwendung und könnten damit potenziell in Kontakt kommen (etwa $0,6 \%$ der Firmen und etwa 0,08\% der Arbeiter des Schweizer Produktionssektors). Mit der Stichprobe wurden nur in wenigen Branchen Nanopartikelanwendungen gefunden: Chemische Industrie, Automobilzulieferer, Elektrotechnik, allgemeiner Handel, Oberflächenbehandlung, Keramik/Glas sowie Steinbehandlung. In weiteren Branchen, wie zum Beispiel Nahrungsmittel, sind zwar Anwendungen bekannt, doch die Verbreitung scheint dort so klein zu sein, dass mit der repräsentativen Stichprobenmenge keine Firma identifiziert wurde.

Die erhobenen Mengen von Nanopartikeln waren in der Regel klein: Nur wenige Firmen wiesen mehr als einige 100 Kilo aus, und dies meist von den bereits fest etablierten Stoffen wie Pigmenten oder Carbon Black.

Es war aber festzustellen, dass in den meisten Betrieben und Branchen zum Schutz von Personen vor allem körpergetragene Schutzausrüstungen eingesetzt wurden (Maske, Handschuhe etc.); technische Lösungen (Einkapselung der Prozesse) wurden seltener genutzt, und auch ein gezielter Schutz der Umwelt (z.B. speziell mit Nanopartikel behandelte oder getrennte Abwässer, Abluft und Abfälle) wurde nur in wenigen Fällen gefunden.

Diese Studie hat einige Fragen zu den vorhandenen Schutzstrategien aufgeworfen, auf welche in Zukunft das Augenmerk gelegt werden sollte. Gleichzeitig hat sie auch aufgezeigt, dass sich heute die Nanopartikelanwendungen nur auf wenige Branchen und dort auf wenige Firmen verteilten und es daher noch nicht zu spät ist, geeignete Schutzmaßnahmen proaktiv zu erforschen, um sie rasch und kostengünstig (sprich: vor Beginn der Hauptproduktion) einführen zu können.

Die Resultate dieser Studie sind national verankert und können nicht uneingeschränkt auf andere Länder übertragen werden, es könnte aber gut sein, dass sich zwischen europäischen Nachbarstaaten der proportionale Anteil von Unternehmen, die Nanopartikel anwenden, nicht allzu stark unterscheidet. $\mathrm{Zu}$ 
bedenken ist jedoch, dass einige der wichtigsten europäischen Produzenten von Nanopartikeln ihren Standort in Deutschland haben.

\section{Wie misst man Nanopartikel?}

Um die Gefährdungssituation eines Arbeiters abschätzen zu können, muss in jedem Fall die Belastung am Arbeitsplatz spezifisch abgeklärt werden. Nanopartikel können vor allem über die Atemluft aufgenommen werden. Eine solche Belastung durch luftgetragene Nanopartikel kann heute mittels verschiedener Messtechniken evaluiert werden. Dabei kommen nachfolgende Aspekte zum Tragen, welche für eine Analyse der Gesundheitsfolgen Wichtigkeit erlangen können.

\section{Masse der Partikel}

Die Masse von Luftpartikeln kann durch die Gewichtszunahme von Filtern bestimmt werden, dies ist aber ein aufwendiges Verfahren. Häufig wird daher das Gewicht von Nanopartikeln approximativ aufgrund der Größenverteilung und der Anzahl der Partikel hochgerechnet. Dabei gilt, je kleiner die Partikel, desto kleiner und unwichtiger ist deren Masse. Die Masse von Nanopartikeln kann dann entscheidend sein, wenn die Partikel löslich sind und die Grundsubstanz toxische Effekte aufweist.

\section{Anzahl der Partikel}

In manchen Fällen zeigt die Anzahl der Nanopartikel eine bessere Korrelation mit toxikologischen Eigenschaften. Große Partikel können durch Streuung von Laserstrahlen detektiert werden, Partikel kleiner als etwa 250 $\mathrm{nm}$ sind aber nicht sichtbar für Laserstrahlen. Zwei Techniken haben sich durchgesetzt, um Partikel kleiner als $250 \mathrm{~nm}$ zu zählen: Partikel können dann optisch gezählt werden, wenn sie durch Anlagerung einer kondensierenden Flüssigkeit soweit vergrößert wurden, dass sie Laserstrahlen streuen. Diesen Trick nutzen die Kondensationskeimzähler (condenzation particle counters: CPC). Daneben gibt es den Nachweis von Nanopartikeln durch das Anlagern von elektrischen Ladungen an die Partikel mit anschließender Messung der Ladungen auf Filtern per Elektrometer (diffusion size classifier). Diese Methode erlaubt einen einfacheren Unterhalt der Geräte, lässt jedoch nur basierend auf mathematischen Modellen Rückschlüsse auf die Zahl der Partikel zu. Die Methode ist gut für Partikel kleiner als $200 \mathrm{~nm}$, nicht jedoch für größere Agglomerate von Nanopartikeln.

\section{Oberfläche der Partikel}

Die Anzahl von Partikeln ist oft nicht genügend, um die biologischen Effekte umfassend erklären zu können. Da chemische Reaktionen vor allem an der Oberfläche von Partikeln ablaufen, wird sie bei reaktiven Partikeln wichtig. Auch die Oberfläche der Partikel kann aus der Anzahl angenähert berechnet werden, diese Berechnung ist aber nur eine grobe Annäherung an die aktive Oberfläche der Partikel. Es gilt weiterhin zu beachten, dass die Oberfläche von Partikeln in der Luft nicht mit derjenigen übereinstimmen muss, welche sie in einer Flüssigkeit aufweisen.

\section{Größenverteilung der Partikel}

Um die Größe von Partikeln zu definieren, wird normalerweise der Größenmittelwert angegeben. Dieser zeigt die dominierende Größe an, aber es gibt immer auch kleinere oder größere Partikel. Die Größe der Partikel ist entscheidend für den Ort der Abscheidung innerhalb der Luftwege [4]. Je kleiner die Partikel, desto tiefer können sie in die Lunge eindringen. Die Größe kann ebenfalls entscheidend sein für die Verbreitung der Partikel innerhalb des Körpers [5] und für das mögliche Eindringen in Zellen [6]. Eine alte Technik für die Ermittlung der Größenverteilung von Partikeln ist die Kaskaden-Impaktion. Kleine Partikel werden dabei von den größeren Partikeln getrennt, weil sie bei kleinen Geschwindigkeiten dem Luftstrom folgen, während größere Partikel an einem Filter abgeschieden werden. Diese getrennte Sammlung auf Filtern erlaubt eine spätere chemische Analyse der Partikel. Die Technik funktioniert mit Einschränkungen auch für 
Nanopartikel, es ist jedoch ein relativ langsames und aufwendiges Verfahren, welches Laborarbeit nach sich zieht.

Vor Ort, und vor allem schneller, werden daher heute die kleinen Partikel aufgrund ihrer unterschiedlichen Mobilität zu großen Partikeln in einem elektrischen Feld und/oder aufgrund ihrer unterschiedlichen Ladungen aufgetrennt. Dies macht die sogenannte differenzielle Mobilitätsanalyse (differential mobility analyzer: DMA). Die Methode kann für die Messung von nicht sphärischen Partikeln ungenau sein, die Annäherung ist aber meist genügend genau. Die zwei Techniken kombiniert (DMA und CPC) erlauben verschiedene Größenklassen von Partikeln zu trennen und so separat zu zählen. Dieses kombinierte System wird dann mit dem englischen Begriff Scanning Mobility Particle Sizer, SMPS, umschrieben. Die Methode eignet sich für Hintergrundsmessungen. Sie erlaubt eine rasche Abschätzung der Größenverteilung von Partikeln in der Luft. Die Methode braucht einige Minuten für eine komplette Analyse, was für kurze Expositionsereignisse zu langsam sein kann. Eine generelle messtechnische Schwierigkeit liegt in der Messung von Agglomeraten, den mehr oder weniger locker zusammengefügten Einzelpartikeln. Für die relativ komplex aufgebauten Partikelagglomerate können die oben genannten Zählmethoden oft nur Schätzungen abgeben.

\section{Fasern}

Es gibt Nanopartikeltypen (Carbon Nanotubes: CNT), die in ihrer Art und auch in ihrer Gesundheitswirkung durch ihre Faserstruktur herausragen. Wenn dieses Material eine Faserlänge von mehr als $3 \mu \mathrm{m}$ aufweist und eine gewisse Steifheit, wie sie bei mehrfachwandigen Kohlenstoffröhrchen auftritt, hat das Immunsystem Mühe, die Partikel geeignet zu eliminieren. Bei diesen speziellen Partikeln könnten asbestähnliche Gesundheitseffekte auftreten [7]. Eine direkt messende Technik, welche einzelne faserstrukturierte Nanopartikel (CNT) spezifisch anzeigen kann, gibt es heute noch nicht. In diesen Fällen muss auf filterbasierte Messungen $\mathrm{zu}$ rückgegriffen werden. Die Filterproben müssen dann unter dem Elektronenmikroskop analysiert werden. Für CNT ist die Filterme- thode aber bisher noch nicht sehr effektiv, hier besteht Entwicklungsbedarf.

Die Vielfalt der Messtechnik und die Schwierigkeiten in der Interpretation der Messdaten zeigt, dass eine einfache Messung mit einem Messgerät oft nicht genügend Informationen für eine abschließende Arbeitsplatzbeurteilung ergibt. Je nach Typ der Exposition und der genutzten Partikel müssen dann tiefergehende Abklärungen erfolgen.

\section{Große Anstrengungen auf Regierungsebene (Schweiz/EU)}

\section{Aktionsplan EU}

Im Aktionsplan der Europäischen Union “Auf dem Weg zu einer europäischen Strategie für Nanotechnologie", einem Bericht der Europäischen Kommission aus dem Jahre 2004 [8], wurden erste Grundlagen gelegt, welche den Schutz von Verbraucher und Arbeitnehmer sowie der Umwelt über den gesamten Lebenszyklus der Produkte einforderten. Der eigentliche Aktionsplan wurde 2005 verabschiedet [9] und die Kommission nahm ihren ersten Durchführungsbericht an zum Aktionsplan für Nanowissenschaften und -technologie 2005 - 2009. Viele Berichte, Mitteilungen, Appelle an die Mitgliedstaaten, Empfehlungen, Überprüfung von Rechtsvorschriften etc. folgten und geben heute eine gute Basis für die Regelungsaspekte bei Nanomaterialien.

\section{Aktionsplan Schweiz}

Im Schweizer Aktionsplan "Synthetische Nanomaterialien"-Bericht des Schweizer Bundesrates April 2008 [10] und dem vorbereitenden Grundlagenbericht [11] wurde bereits festgehalten, dass die Art der verwendeten Nanomaterialien und die angewandten Schutzmaßnahmen die Exposition stark beeinflussen und dass diesbezüglich noch viele Fragen offen sind. Bei der Vielfalt von Einflussfaktoren und einer noch größeren Vielfalt von Partikeltypen kann keine einfache Regel aufgestellt werden, von welchen Partikeln Gesundheitsauswirkungen erwartet 
werden und von welchen nicht. Aber gewisse grobe Richtlinien können helfen, mindestens die gefährlicheren aufzuzeigen.

\section{Schweizer Vorsorgeraster}

Ein erstes Produkt des schweizerischen Aktionsplans ist der "Vorsorgeraster für Synthetische Nanomaterialien" [12] vom Bundesamt für Gesundheit und Bundesamt für Umwelt.

Das Raster soll Industrie und Gewerbe ein strukturiertes Vorgehen zum Erkennen von Risiken im Umgang mit synthetischen Nanomaterialien ermöglichen. Es erlaubt das Risikopotenzial eines synthetischen Nanomaterials und dessen Anwendungen für Arbeitnehmer, Verbraucher und die Umwelt in strukturierter Weise mit einem einfachen Screening aufgrund des heute vorhandenen Wissens abzuschätzen. Es zeigt auf, wann weitergehende Abklärungen nötig sind.

\section{Empfehlungen der Internationalen Organisation für Normung (ISO)}

Die Internationale Organisation für Normung, ISO, hat 2005 ein technisches Komitee gegründet, um Ordnung in die Begrifflichkeit rund um die Nanotechnologie zu bringen. Eine solche Nomenklatur wurde 2008 veröffentlicht [1]. Erste Vorbereitungen für Standards innerhalb von Messgeräten, Methoden, Modellen, Simulationen sowie eine erste Sammlung von Gesundheits- und Sicherheitsregeln im Arbeitsbereich wurden als Informationssammlung im ISO/TR 12885 vereint [13].

\section{Empfehlungen nationaler Gremien}

\section{Schweiz}

Die Schweizerische Unfall Versicherungs-Anstalt (SUVA) stellt seit mehreren Jahren auf ihrer Homepage Empfehlungen zur Verfügung, welche den angemessenen Umgang mit Nanopartikeln beschreibt [14].
Die Informationen werden dem aktuellen Wissensstand laufend angepasst, sind jedoch im Kern seit Beginn gleich geblieben und lauten grob zusammengefasst folgendermaßen: Die Nanopartikel stellen ein potenzielles Gesundheitsrisiko dar. Das Ausmaß der gesundheitlichen Risiken kann zurzeit noch nicht schlüssig bewertet werden. Es empfiehlt sich daher, im Sinne der Prävention, die Exposition gegenüber Nanopartikeln zu minimieren. Das Vorgehen dafür ist generell gleich wie zum Schutz vor anderen gefährlichen Stoffen, in hierarchischer Ordnung:

- Substitution: Ersetzen von gesundheitsgefährdenden Stoffen durch harmlosere.

- Kollektivschutz: Technische Maßnahmen zum Erfassen, Begrenzen und Abführen gefährlicher Gase, Dämpfe und Stäube.

- Individualschutz: Verwendung persönlicher Schutzausrüstung zusätzlich zu den technischen Maßnahmen.

- Hygiene: Geeignete Waschgelegenheiten, geschützte Aufbewahrung der nicht beruflich eingesetzten Kleider ("Ausgangskleider”).

\section{Deutschland}

Die Bundesanstalt für Arbeitsschutz und Arbeitsmedizin (BAuA) hat zusammen mit dem Verband der Chemischen Industrie (VCI) einen "Leitfaden für Tätigkeiten mit Nanomaterialien am Arbeitsplatz" erstellt [15], in welchem nebst derzeitigen Empfehlungen und Handlungsanweisungen auch der Stand der Messtechnik nachzulesen ist. Zusätzlich wurden auch die Ergebnisse einer Firmenbefragung veröffentlicht, welche die aktuellen Schutzstrategien in einigen deutschen Firmen evaluierte, die mit Nanopartikel arbeiten.

\section{Aktuelle Herausforderungen}

Die heutige Herausforderung sowohl für nationale Organisationen wie auch für die Forschung ist es herauszufinden, welche die besten Präventionsstrategien sind und in welchen Bereichen der Industrie diese am erfolgreichsten angewendet werden können. Grobstrategien müssen gezielt auf die Bedürfnisse von Firmen abgestimmt werden und diese da- 
bei unterstützen, eine sichere Nutzung von Nanomaterialien zu entwickeln. Insbesondere kleine und mittlere Unternehmen (KMU) brauchen häufig Unterstützung in der Umsetzung von Schutzmaßnahmen. Je früher Schutzmaßnahmen für Personal und Umwelt entwickelt und eingesetzt werden, desto kosteneffizienter sind sie gewöhnlich.

Für eine abschließende Beurteilung der Gesundheitsrisiken fehlen heute noch viele Informationen. Daher müssen jetzt die Datengrundlagen geschaffen werden für zukünftige Messkampagnen, quantitative Risikoanalysen und epidemiologische Studien. Wenn heute größere Gesundheitsstudien noch nicht gestartet werden können, dann sollte zumindest Gewicht auf die Dokumentation von genutzten Materialien und potenziellen Expositionen gelegt werden.

\section{Schlussfolgerung}

Die Nanotechnologie hat mit ersten einfacheren Materialien in der Industrie Fuß gefasst, weitere Anwendungen sind geplant. Die Anwendungen sind heute oft noch im Versuchsstadium und werden sich erst in den kommenden Jahren im großen Stil entwickeln. Es ist absehbar, dass die Frage nach Gesundheitsauswirkungen von Nanomaterialien immer öfters an Arbeitsmediziner und -hygieniker herangetragen werden wird. Für eine kompetente Beratung ist wichtig, dass sich die verantwortlichen Personen über die Forschung zu gesundheitlichen Effekten von Nanopartikeln auf dem Laufenden halten und welche spezifischen Mess- und Schutzmethoden zur Verfügung stehen.

\section{Literatur}

[1] ISO/TC 229 - Nanotechnologies. Nanotechnologies - terminology and definitions for nanoparticles. ISO/TS 27687; 2008(E).

[2] Woodrow Wilson International Center for Scholars. Project on Emerging Nanotechnologies "A Nanotechnology Consumer Products Inventory". www.nanotechproject.org/inventories/consumer, 2007 (13. März 2009).

[3] Schmid K, Riediker M, Danuser B. "Swiss nanoinventory": an assessment of the usage of nanoparticles in Swiss industry: final report. Lausanne: Institute for Work and Health; 2008.

[4] ICRP. Human Respiratory Tract Model for Radiological Protection. Annals of the ICRP, Publication
66 ed. New York: International Commission on Radiological Protection; 1994.

[5] Kreyling WG, Semmler M, Erbe F, Mayer P, Takenaka S, Schulz $H$ et al. Translocation of ultrafine insoluble iridium particles from lung epithelium to extrapulmonary organs is size-dependent but very low. J Toxicol Environ Health A. 2002; 65: 1513-1530.

[6] Geiser M, Rothen-Rutishauser B, Kapp N, Schurch S, Kreyling W, Schulz H et al. Ultrafine particles cross cellular membranes by non-phagocytic mechanisms in lungs and in cultured cells. Environ Health Perspect. 2005; 113: 1555-1560.

[7] Poland CA, Duffin R, Kinloch I, Maynard A, Wallace WAH, Seaton A et al. Carbon nanotubes introduced into the abdominal cavity of mice show asbestos-like pathogenicity in a pilot study. Nature Nanotechnology. 2008; 3: 423-428.

[8] Europäische Kommission. Nanowissenschaften und Nanotechnologien: Aktionsplan für Europa 2005 - 2009. ftp://ftp.cordis.europa.eu/pub/nanotechnology/docs/action_plan_brochure_de.pdf, 2005 (13. März 2009).

[9] European Commission. Nanosciences and nanotechnologies: communication from the commission to the council, the European parliament and the economic and social committee: an action plan for Europe 2005 - 2009. http://cordis.europa. eu/nanotechnology/actionplan.htm, 2005 (13. März 2009); COM(2005) 243 final.

[10] Federal Office of Public Health FOPH, State Secretariat for Economic Affairs SECO, Federal Office for the Environment FOEN. Swiss action plan on "Risk Assessment and Risk Management for Synthetic Nanomaterials 2006 - 2009". www.environment-switzerland.ch/div-4002-e, 2009 (13. März 2009).

[11] Meili C, Widmer M, Husmann F, Gehr P, Blank F, Riediker $M$ et al. Synthetische Nanomaterialien. Risikobeurteilung und Risikomanagement. Grundlagenbericht zum Aktionsplan. Umwelt-Wissen Nr. 0721 ed. Bundesamt für Umwelt und Bundesamt für Gesundheit; 2007.

[12] Federal Office of Public Health FOPH, Federal Office for the Environment FOEN. Precautionary matrix for synthetic nanomaterials. http://www. bag.admin.ch/themen/chemikalien/00228/00510/ 05626/index.html, 2009 (13. März 2009).

[13] ISO/TC 229 - Nanotechnologies. ISO/TR 12885: Nanotechnologies - health and safety practices in occupational settings relevant to nanotechnologies, 2008

[14] SUVA. Guidelines for safe applications of nanoparticles. http://www.suva.ch/nanopartikel, 2009 (11. März 2009).

[15] $B A u A / V C I$. Leitfaden für Tätigkeiten mit Nanomaterialien am Arbeitsplatz. http://www.baua.de/nn 44628/de/Themen-von-A-Z/Gefahrstoffe/Nanotechnologie/pdf/Leitfaden-Nanomaterialien.pdf, 2007 (13. März 2009).

K. Schmid, Dipl. Umwelt Natw. ETH Institut für Arbeit und Gesundheit

Rue du Bugnon 21

CH-1011 Lausanne

e-mail: Kasparschmid@alumni.ethz.ch 